\title{
Utilizing Atlas-Based Modeling to Predict Knee Joint Cartilage Degeneration: Data from the Osteoarthritis Initiative
}

\author{
Mika E. Mononen (i), ${ }^{1}$ Mimmi K. Liukkonen, ${ }^{1}$ and Rami K. Korhonen ${ }^{1,2}$ \\ ${ }^{1}$ Department of Applied Physics, University of Eastern Finland, POB 1627, 70211 Kuopio, Finland; and ${ }^{2}$ Diagnostic Imaging \\ Centre, Kuopio University Hospital, Kuopio, Finland
}

(Received 29 May 2018; accepted 5 December 2018; published online 13 December 2018)

Associate Editor Michael S. Detamore oversaw the review of this article.

\begin{abstract}
Currently, there are no clinically available tools or applications which could predict osteoarthritis development. Some computational models have been presented to simulate cartilage degeneration, but they are not clinically feasible due to time required to build subject-specific knee models. Therefore, the objective of this study was to develop a template-based modeling method for rapid prediction of knee joint cartilage degeneration. Knee joint models for 21 subjects were constructed with two different template approaches (multiple templates and one template) based on the MRI data. Geometries were also generated by manual segmentation. Evaluated volumes of cartilage degeneration for each subject, as assessed with the degeneration algorithm, were compared with experimentally observed 4 year follow up Kellgren-Lawrence (KL) grades. Furthermore, the effect of meniscus was tested by generating models with subjectspecific meniscal supporting forces and those with the average meniscal supporting force from all models. All tested models were able to predict most severe cartilage degeneration to those subjects who had the highest KL grade after 4 year follow up. Surprisingly, in terms of statistical significance, the best result was obtained with one template approach and average meniscal support. This model was fully able to categorize all subjects to their experimentally defined groups (KL0, KL2 and KL3) based on the 4 year follow-up data. The results suggest that a template- or population-based approach, which is much faster than fully subject-specific, could be applied as a clinical prediction tool for osteoarthritis.
\end{abstract}

Keywords-Knee joint, Articular cartilage, Osteoarthritis, Finite element analysis, Degeneration, Template modeling.

Address correspondence to Mika E. Mononen, Department of Applied Physics, University of Eastern Finland, POB 1627, 70211 Kuopio, Finland. Electronic mail: mika.mononen@uef.fi

\section{INTRODUCTION}

Computational finite element (FE) models of the knee joint are able to offer a quantitative estimation about risks for the onset and development of knee osteoarthritis (OA) based on mechanical signals experienced by tissues. ${ }^{18,19,40}$ They can also be used to assess the feasibility of different rehabilitation and surgical protocols. These models are typically based on subject-specific knee joint geometries with varying types of loading conditions. Physiologically relevant loadings have also been implemented, such as gait. ${ }^{1,4}$ Recently developed knee models with a cartilage degeneration algorithm were even able to predict mechanical changes of articular cartilage during the development of OA in a time-dependent manner. ${ }^{28,32}$

The main reason why the aforementioned models have not been utilized in clinical purposes is related to time needed to generate the models. Especially manual segmentation to generate knee geometries is timeconsuming. There has been evolution in segmentation methods toward automatization. 2,24,27,37 However, these methods are typically critically dependent on the image quality, and manual work is still needed after the initial segmentation. For instance, automated segmentation methods may cause easily inaccuracies (roughness) in contacting surfaces. This leads to difficulties in generation of proper FE meshes and causes also convergence problems.

Generation of proper FE meshes for tissues and contact interactions may be even more time-consuming challenge compared with the time required for segmentation. Especially models that include several tissues in contact with each other, complex loading conditions, and large tissue deformations are critically dependent on the mesh quality. Currently, there is one approach available to generate automatic FE meshes 
for soft tissues in the knee joint. ${ }^{33}$ However, even in this highly promising approach the mesh is geometryspecific and always different which could again cause challenges in complex contacts and simulations.

To save time in generation of model geometries and proper FE meshes, a template-based FE modeling may offer a solution. In template approaches, an existing template (geometry of a certain tissue, i.e., atlas) is deformed to match certain anatomical landmarks/dimensions determined either manually or automatically from clinical images. ${ }^{9,16}$ Interestingly, current literature does not include any attempts to apply template modeling to evaluate knee joint cartilage mechanics and subject-specific onset and progression of knee OA. Easy implementation of feasible loading conditions and meniscal load transfer are also desired features if rapid model generation and simulation are of interest. ${ }^{6,7,12-14}$

Without considering knee injuries, two main risk factors for $\mathrm{OA}$ are overweight and aging. ${ }^{38,44}$ Currently, there are no computational studies available where these parameters would have been considered simultaneously for the prediction of subject-specific onset and development of OA. In computational FE models, the weight of a subject can be simply considered by the boundary conditions. However, there have been no attempts to consider aging. Though, the mechanisms behind $\mathrm{OA}$ due to aging can be very complex, ${ }^{29,38}$ it could be considered in the model simply by changing tissue material properties with age according to experimental evidence. ${ }^{20}$

In the current study, the main aim is to introduce a workflow for template-based modeling of the knee joint and show its potential to predict the development of knee OA. In addition to rapid generation of knee geometries and FE meshes, the template model includes automatic implementation of tissue material properties and boundary conditions. It also utilizes the previously developed cartilage degeneration algorithm to predict the development of $\mathrm{OA}^{26,31}$ and considers aging. Model predictions are compared against experimentally observed radiographic Kellgren-Lawrence follow-up data and subject-specific FE models.

\section{METHODS}

\section{Subject Information}

Data used in the preparation of this article were obtained from the Osteoarthritis Initiative (OAI) database, which is available for public access at http://oa i.epi-ucsf.org/. Specific datasets used are AllClinical00, AllClinical06, 0.C.2 0.E.1, 6.C.1, 6.E.1, kxr_sq_bu00 and kxr_sq_bu06. The same experimental data used
FIGURE 1. Workflows for the patient-specific (top) and template (bottom) approaches. In the patient-specific approach, the model geometry (whole knee joint) is generated via manual segmentation and then the simplified gait load assumed to occur during the stance phase of the gait is applied. Next, boundary conditions and forces from the whole knee joint simulation are used as an input in the compartment model. In the compartment model, the effect of meniscus is considered by subtracting forces through the meniscus from the total joint loads in the compartment (see variation of forces through menisci from Fig. 4). Finally, cartilage degeneration in the compartment model is simulated with the degeneration algorithm [Eq. (10)]. In the template approaches, anatomical dimensions (AD) are first measured from all subjects $(N=21)$ based on the MRI data (see details from Fig. 2). Then, the matching template model for the subject of interest is found based either on the minimum RMSE of ADs between the subject of interest and templates (multiple templates) or the minimum RMSE of ADs between each subject and all templates (one, best-matching template is chosen). Next, the optimal template model is scaled to match AD's for the subject of interest. Finally, the scaled template model is simulated together with the simplified gait loading ( $50 \%$ of the total joint loads assumed to occur in the medial compartment) and the degeneration algorithm [Eq. (10)]. The effect of meniscus is considered identically with the manual approach.

previously was applied here. ${ }^{26}$ Baseline and 4-year follow-up MRIs and radiographic KL grades were collected from 21 subjects (8 males and 13 females). Ages and weights of subjects were obtained from the baseline visit. Subjects were divided into three groups based on the baseline weight and the experimentally observed 4-year follow up KL grade (KL0 (BMI = $\left.23.1 \pm 1.6 \mathrm{~kg} / \mathrm{m}^{2}\right), \operatorname{KL} 2\left(\mathrm{BMI}=33.0 \pm 2.2 \mathrm{~kg} / \mathrm{m}^{2}\right)$ and $\mathrm{KL} 3\left(\mathrm{BMI}=33.7 \pm 3.7 \mathrm{~kg} / \mathrm{m}^{2}\right), 7$ subjects in each group). At the baseline, KL grade was zero (indicating no signs of $\mathrm{OA}$ ) in each group, whereas at 4-year follow-up, KL grades for KL0, KL2 and KL3 groups were 0 (intact), 2 (doubtful OA) and 3 (severe $\mathrm{OA}$ ), respectively. The subjects were selected in order to show that in addition to mechanical overloading (overweight subjects in KL2 and KL3 groups), other factors, such as subject-specific knee joint geometries, can have a contribution in the initiation and development of cartilage degeneration. Specific exclusion criteria are seen from a previous study where the same subjects were used. ${ }^{26}$ Ethical approval and informed consent for collecting all subject information was provided by the OAI. The present project has been reviewed by a local data protection officer, and the local approval to conduct the study was granted by the University of Eastern Finland Committee on Research Ethics.

\section{Patient-Specific Approach}

Since the patient-specific approach with manual phases in the model generation is not new, here it is 


\section{Workflow - Subject-specific approach [1]}

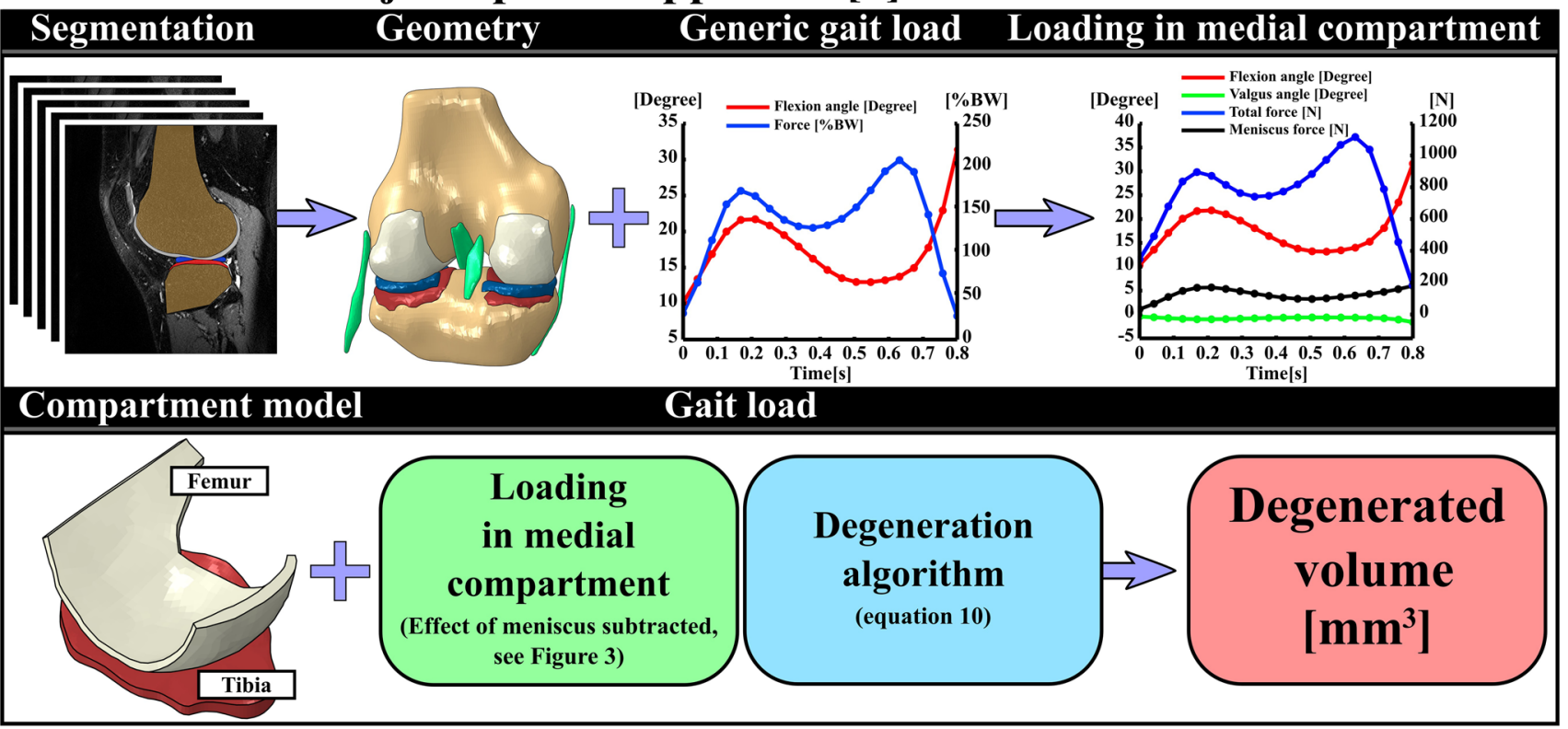

\section{Workflow - Template approach}

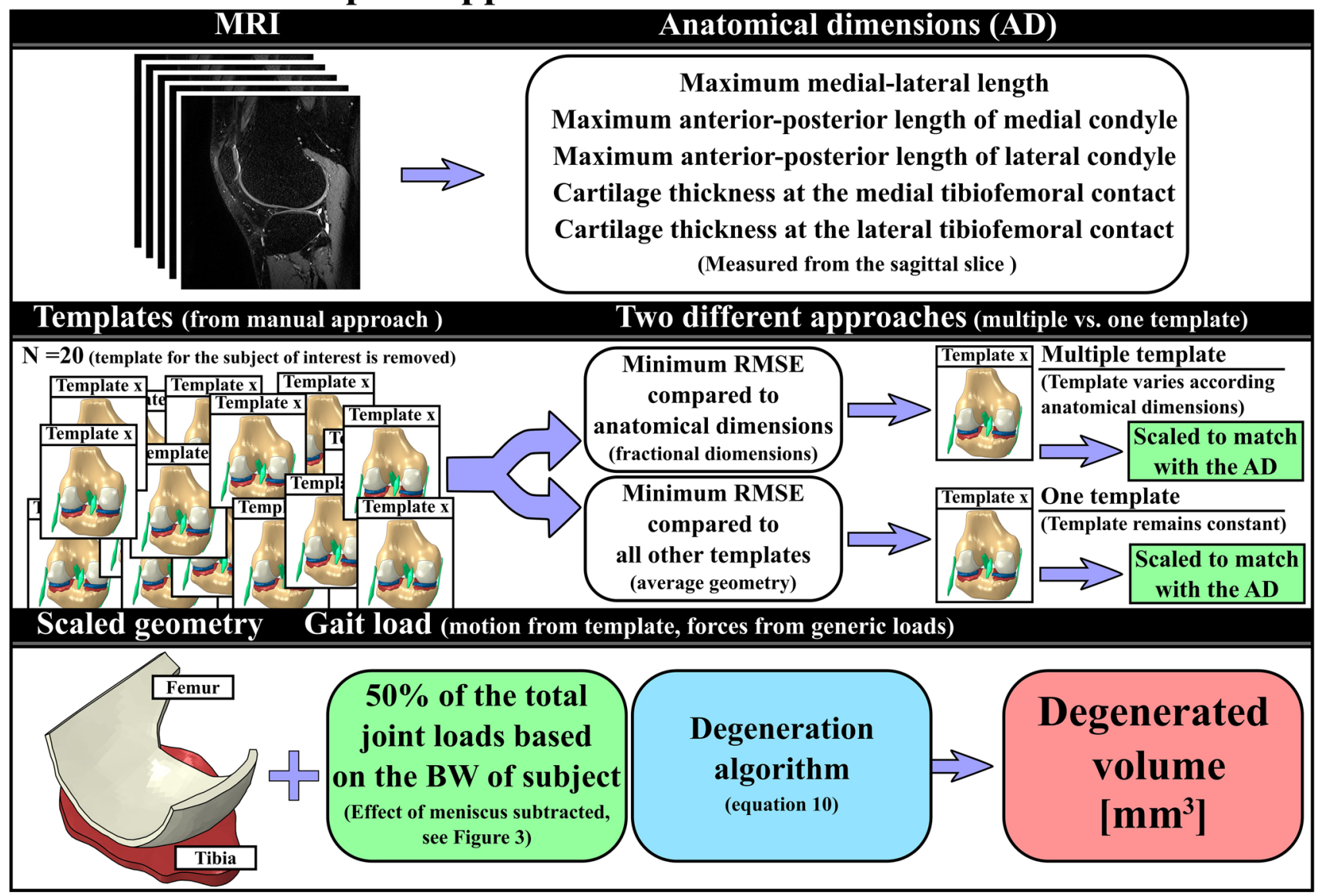


described only briefly. Knee joint geometries and FE meshes of cartilages and menisci were utilized directly from our previous study. ${ }^{26}$ Then, each knee joint was exposed to physiologically relevant loading, ${ }^{8,23} \mathrm{mim}$ icking simplified walking (Fig. 1). The flexion angle and joint reaction force (scaled by subject weight in bodyweight) were implemented in the model as a function of stance. In order to produce smooth contact between medial and lateral tibiofemoral compartments, the varus-valgus rotation was set free, while other degrees of freedoms were fixed (Fig. 1, Patientspecific approach-top row).

In order to have accurate predictions within a reasonable time, compartment models with finer meshes were generated. The compartment models were run by the outputs from the whole knee joint models (Fig. 1). In the compartments models, the load transfer of meniscus was subtracted from the total reaction forces through the compartment. This simplification may affect the simulation results, especially during complex loading conditions. However, as demonstrated in a previous study, ${ }^{28}$ this assumption was shown to have a negligible effect on the simulated stress, contact pressure and pore pressure levels at the tibiofemoral contact. See detailed description of these different model generation phases from a previous study. ${ }^{26}$ Implementation of the materials and the degeneration algorithm have been described below.

\section{Template Approach}

First, morphological dimensions (anterior-posterior, AP and medial-lateral, ML) of distal femur and tibiofemoral joint space width (JSW, sum of total cartilage thicknesses from femoral and tibial cartilage) were measured from each knee joint based on the MRI data (Fig. 1, Template approach and Fig. 2). The ML dimension was determined for clinical transepicondylar axis. The AP dimensions were determined for the lines producing the maximum anterior-posterior distance for the ellipse-like shaped medial and lateral condyles. Cartilage thicknesses (JSWs) were obtained from both medial and lateral compartments, measured at the central locations of tibiofemoral contact regions. Each dimension was determined from sagittal images. To study the influence of different joint shapes based on anatomical dimensions, ${ }^{21}$ two different template approaches were tested:

(1) Multiple templates (Best corresponding individual template) Morphological dimensions and cartilage thicknesses were first normalized with respect to the ML dimensions in each template, similarly as in earlier studies. ${ }^{21,35}$ The most suitable tem-

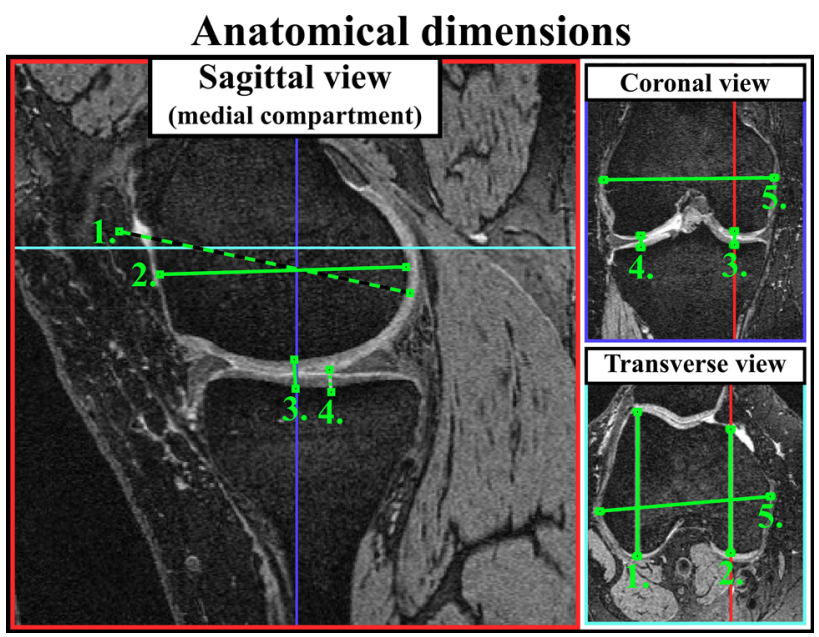

FIGURE 2. Determining anatomical dimensions. Number 1 is the maximum anterior-posterior length of lateral femoral condyle, number 2 is the maximum anterior-posterior length of medial femoral condyle, number 3 is the medial tibiofemoral cartilage thickness, number 4 is the lateral tibiofemoral cartilage thickness, and number 5 is the maximum medial-lateral distance of distal femur (clinical transepicondylar axis). All measurements were measured based on the sagittal view.

plate from 21 geometries (meshes) was selected based on the smallest root mean square error (RMSE) in the normalized morphological dimensions between the current subject under investigation and templates. While calculating the RMSE, the template for the current subject was omitted from the template library. Finally, the chosen template model geometry was scaled to match exactly with the morphological dimensions of the current subject.

(2) One template (Best from 21 templates) Based on the normalized morphological dimensions, the sum of the RMSE was calculated between each subject and all other subjects. Then, those normalized subject dimensions that produced the smallest sum of RMSE was considered to represent average morphological joint dimensions and geometry. Finally, the model geometry from that one single template was scaled to match with morphological dimensions of each subject.

In both template methods, the scaling was based on the cartesian coordinate system. Each template was positioned so that AP, ML and tibiofemoral cartilage thickness directions corresponded to cartesian axes $(x$, $y, z)$. The scaling was considered by multiplying the nodal values of the best template with percentage differences in ML, AP and cartilage thickness directions $(x, y, z)$ between the subject of interest and the best template (Fig. 3). 


\section{The best matching template}

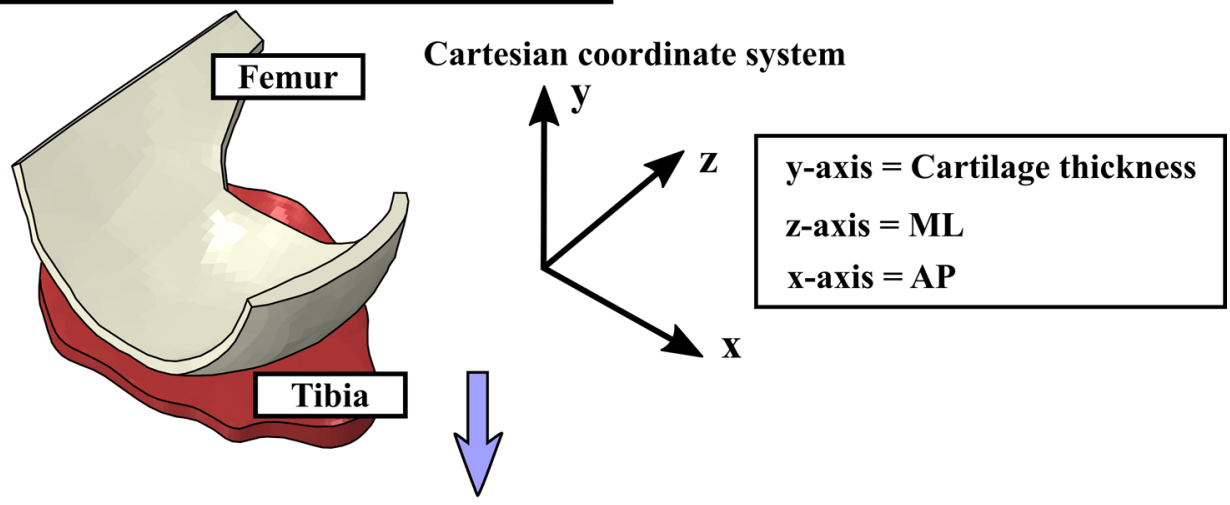

Scaling of the template (difference between the best template and the subject of interest)
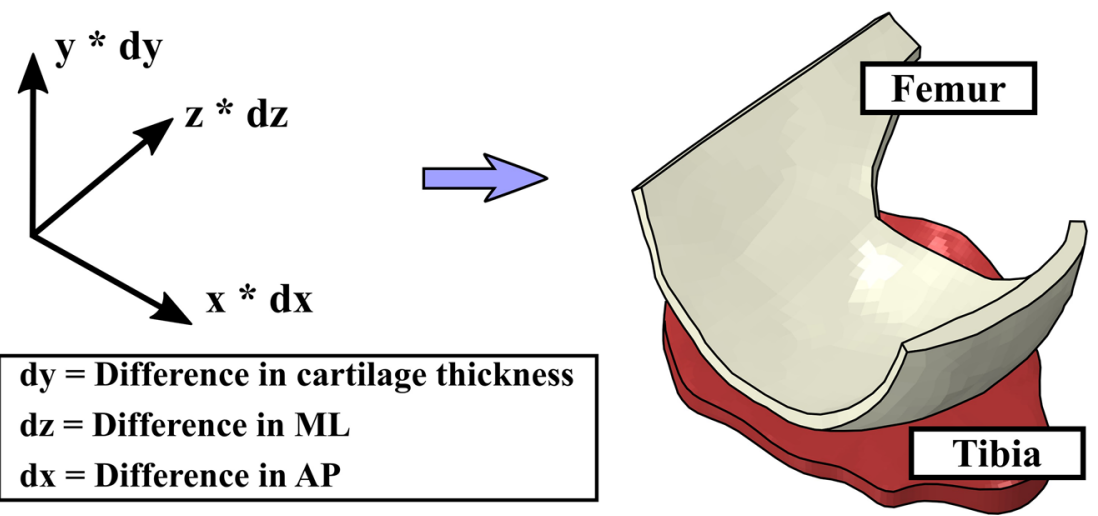

FIGURE 3. Scaling of the template. Nodal values in the best matching template model are multiplied with percentage differences in the measured ML, AP and cartilage thickness directions $(d x, d y, d z)$ between the subject of interest and the best template.

In this template approach, in order to speed up the model generation and simulation process, the whole knee joint was not simulated. Instead, only the medial compartment was considered in further simulations. The flexion angle and varus-valgus rotation were obtained from the best matching template (Fig. 1, subject-specific approach-loading in the medial compartment), whereas the joint reaction force was considered with $50 \%$ of the total knee joint forces during gait loading based on the bodyweight of the subject of interest. ${ }^{22}$ The effect of meniscal support was considered similarly as in the patient-specific approach. This produced a good match in joint reaction forces through the medial compartment between patient-specific and template approaches. Implementation of the materials and the degeneration algorithm have been described below.

\section{Material Models and Cartilage Degeneration Algorithm}

The following phases in the model generation and simulation were done similarly in the patient-specific and template approaches. Based on previous studies, cartilage and meniscus were considered as fibril reinforced poroviscoelastic (FRPVE) and transverse isotropic elastic materials, respectively. ${ }^{39,41,42,45}$ See the material properties from Table 1. Though meniscus is a poroelastic material, it could be assumed that only negligible fluid flow would occur during fast loading conditions. Thus, the effect of fluid in meniscus was omitted.

Osteoarthritic changes have been reported to be more frequently in the medial than lateral compartment. ${ }^{5,43}$ Also these patients developed OA in the medial compartment. ${ }^{26}$ For these reasons, only cartilage degeneration in the medial compartment was simulated. Based on the previous experimental studies, ${ }^{17,30,34,36}$ cartilage degeneration may be initiated and progressed due to excessive and cumulatively accumulated tissue tensile stresses experienced primarily by the collagen fibril network. In our previous studies, ${ }^{26,31}$ this was considered iteratively by degenerating the collagen fibril network as follows:

$$
D_{e l, i}=1, \quad \text { if }(i=1),
$$


TABLE 1. Material parameters of the FPRVE material model for the femoral and tibial cartilages and the transverse isotropic elastic material for the meniscus.

\begin{tabular}{llll}
\hline Parameter & Femoral cartilage & Tibial cartilage & Meniscus \\
\hline Collagen fibril network architecture & Depth-wise arcade-like & Depth-wise arcade-like \\
$E_{\mathrm{m}}(\mathrm{MPa})$ & 0.215 & 0.106 & - \\
$E_{0}(\mathrm{MPa})$ & 0.92 & 0.18 & - \\
$E_{\varepsilon}(\mathrm{MPa})$ & 150 & 23.6 & - \\
$v_{\mathrm{m}}$ & 0.15 & 0.15 & - \\
$\eta(\mathrm{MPa} \mathrm{s})$ & 1062 & 1062 & - \\
$k\left(10^{-15} \mathrm{~m}^{4} / \mathrm{Ns}\right)$ & 6 & 18 & - \\
$n_{\mathrm{f}}$ & $0.8-0.15^{\star} h z$ & $0.8-0.15^{\star} h z$ & - \\
$T_{\sigma_{f}}(\mathrm{MPa})$ & Eq. $(10)$ & Eq. $(10)$ & - \\
$E_{1}, E_{2}(\mathrm{MPa})$ & - & - & - \\
$E_{3}(\mathrm{MPa})$ & - & - & 20 \\
$v_{12}$ & - & - & 0.3 \\
$v_{31}$ & - & - & 0.78 \\
$G_{13}(\mathrm{MPa})$ & - & - & 50 \\
\hline
\end{tabular}

All parameters are based on the previous studies. ${ }^{15,39,45}$

$h z$ normalized depth of the tissue, $E_{\mathrm{m}}$ non-fibrillar matrix modulus, $E_{0}$ initial fibril network modulus, $E_{\varepsilon}$ strain-dependent fibril network modulus, $v_{\mathrm{m}}$ Poisson's ratio of the non-fibrillar matrix, $\eta$ viscoelastic damping coefficient of fibrils, $k$ permeability, $n_{\mathrm{f}}$ fluid fraction, $T_{\sigma_{\mathrm{f}}}$ age dependent degeneration threshold, $E_{1}, E_{2}, E_{1}$ radial, axial and circumferential Young's moduli, respectively, $v_{12}, v_{31}$ Poisson's ratios, $G_{13}$ shear modulus.

$$
\begin{aligned}
& D_{e l, i}=D_{e l, i-1}-\left(D_{e l, i-1} \sqrt[1.5]{\sum_{t=1}^{T O T} D_{e l, t} \times I N C_{t}}\right) \\
& \quad \operatorname{if}(i>1)
\end{aligned}
$$

where, $D_{e l, i}$ is the current degeneration level $(1=$ no degeneration; $0=$ total degeneration) inside a specific element (el) after certain iteration $(i), I N C_{t}$ is the duration of each time increment, TOT is the total number of required time points $(t)$ during each iterative loading step, and $D_{e l, t}$ is the fibril degeneration factor. The degeneration equations [Eqs. (1) and (2)] were applied during the stance phase of the gait with the fibril degeneration factor $\left(D_{e l, t}\right)$, which was calculated for each element $(e l)$ at each time point $(t)$ using as follows:

$$
\begin{gathered}
D_{e l, t}=\left(\frac{\left(S_{e l, t}-T_{\sigma_{\mathrm{f}}}\right) / T_{\sigma_{\mathrm{f}}}}{100}\right), \quad \text { if }\left(S_{e l, t}>T_{\sigma_{\mathrm{f}}}\right), \\
D_{e l, t}=0, \quad \text { if }\left(S_{e l, t} \leq T_{\sigma_{\mathrm{f}}}\right),
\end{gathered}
$$

where $S_{e l, t}$ is the tensile stress for each element at each time point during the stance phase of the gait and $T_{\sigma_{\mathrm{f}}}$ is the threshold value for cartilage damage. In this previous study, the iterative process reduced the collagen network stiffness as a function of time, simulating changes in $\mathrm{OA}^{26,31}$ In the present, this approach was modified (see below).

\section{Aging}

The link between aging and increased probability for the cartilage degeneration was considered by the experimentally observed relation between age and tensile failure limit for cartilage tissue. ${ }^{20}$ Thus, the following equations are proposed to link age and the threshold for the initiation and development of cartilage degeneration:

$$
\begin{aligned}
& T_{\sigma_{\mathrm{f}}}=30 \mathrm{MPa}, \quad \text { if }(\text { Age }<30), \\
& T_{\sigma_{\mathrm{f}}}=30 \mathrm{MPa}-((\text { Age }-30)(20 / 15)), \\
& \quad \text { if }(30 \leq \mathrm{Age} \leq 45), \\
& T_{\sigma_{\mathrm{f}}}=10 \mathrm{MPa}-((\mathrm{Age}-45)(3 / 20)) \\
& \quad \text { if }(45<\mathrm{Age} \leq 65), \\
& T_{\sigma_{\mathrm{f}}}=7 \mathrm{MPa}-((\text { Age }-65)(2 / 100)) \\
& \quad \text { if }(65<\mathrm{Age} \leq 75), \quad \text { if }(\text { Age }<75), \\
& T_{\sigma_{\mathrm{f}}}=6.8 \mathrm{MPa}, \quad
\end{aligned}
$$

These thresholds were then used with Eqs. (1)-(4) to simulate cartilage degeneration. To provide degeneration evaluations within reasonable times, which is again important for clinical purposes, iterative interphases from Eq. (2) were removed and final predictions were based only on the tissue tensile stresses after one simulation. Only degenerated volumes were analyzed, i.e., information about elements where $D_{e l, i}$ was 
reduced was sufficient. This was an acceptable simplification, because time in Eq. (2) is arbitrary and the predicted degeneration volume was shown to be only slowly progressive (over $70 \%$ of total volumetric degeneration $\left(D_{e l, i}<1\right)$ occurred after the first iteration in subjects with substantial volumetric degeneration after 100 iterations). ${ }^{26}$ Based on this assumption, iterations (i) were replaced with aging (Age). Thus, Eqs. (1) and (2) were merged into the following formation:

$$
D_{e l, \mathrm{Age}}=1-\left(\sqrt[1.5]{\sum_{t=1}^{\mathrm{TOT}} D_{e l, t} \times I N C_{t}}\right)
$$

where $D_{e l, \text { Age }}$ is indicating element-wise cartilage degeneration and Age refers to the subject age.

\section{Meniscus}

First, by the subject-specific approach, the forces through the medial and lateral menisci of the whole knee joint models were simulated for each subject. Based on these simulations, input data for the models with subject-specific and average meniscus support were produced and they could be used as an input in further analysis of the compartment modes in patientspecific and template approaches. The average meniscus support was calculated separately as a fraction of total forces through tibiofemoral contact for the medial and lateral menisci. In computational consideration of meniscus, subtraction of load transfer of meniscus from the total reaction forces through the compartment was justified in a previous study. ${ }^{28}$ That study demonstrated identical cartilage responses between knee joint models with menisci and those with menisci considered as described above.

\section{Simulations}

All FE simulations were performed with Abaqus FE package (v6.13-3, Dassault Systèmes, Providence, RI, USA) using UMAT subroutine to implement FRPVE material properties for cartilage tissues. Before comparing patient-specific and template approaches, volumetric degeneration predictions based on the Eqs. (1), (2) and (10) were compared with each other, because that parameter has been shown to correlate well with the predicted level of OA. ${ }^{26}$ For this comparison, a test subject was selected based on the preliminary simulations where severe cartilage degeneration was expected. It was noticed that volumetric degenerations matched between the Eqs. (1), (2) and (10). Then, patient-specific and template approaches were introduced for each subject and pre- dictions for the development of knee OA were simulated with the Eq. (10) for the following approaches:

(1) Subject-specific approach 1-subject-specific geometry with subject-specific meniscus support

(2) Subject-specific approach 2-subject-specific geometry with average meniscus support

(3) Multiple templates 1-the best template with subject-specific meniscus support

(4) Multiple templates 2-the best template with average meniscus support

(5) One template 1 - average template with subjectspecific meniscus support

(6) One template 2 - average template with average meniscus support

In each simulation, cartilage degeneration [Eq. (10)] was based on the maximum principal (tensile) tissue stresses during physiologically relevant loading conditions, mimicking the stance phase of the gait. ${ }^{8,23} \mathrm{Fi}$ nally, degenerated volumes were determined for each subject and those were compared between different cases (see above).

\section{Statistical Analysis}

Statistical differences between KL grade groups and predicted degeneration volumes were analyzed with the Kruskal-Wallis $H$ test. Predictive accuracy of the algorithm was tested using receiving operating characteristics (ROC) and area under curve (AUC). ${ }^{25}$ The level of significance was set to $p<0.05$. Statistical analysis was performed using SPSS software (SPSS 23.0, SPSS Inc., Chicago, IL, USA).

\section{RESULTS}

In the subject-specific approach, time from the model generation till the final prediction for one subject was at minimum of 1 week. This time was reduced to $\sim 2 \mathrm{~min}$ in the template approach.

In the analysis of the whole knee joint models, it was noticed that the meniscus support force was highly subject-specific at both the medial and lateral compartments (Fig. 4). The ranges of maximum and average support in the medial compartment were 0-35 and $6-25 \%$ of the total knee joint forces, respectively (Fig. 4, left). The corresponding values at the lateral compartment were $0-37$ and $5-11 \%$, respectively (Fig. 4, right).

In each patient-specific and template-based model (1-6), KL2 and KL3 groups produced higher degenerated volumes compared to KL0 group (Fig. 5). In the subject-specific approach with both subject-specific 


\section{Meniscal support}
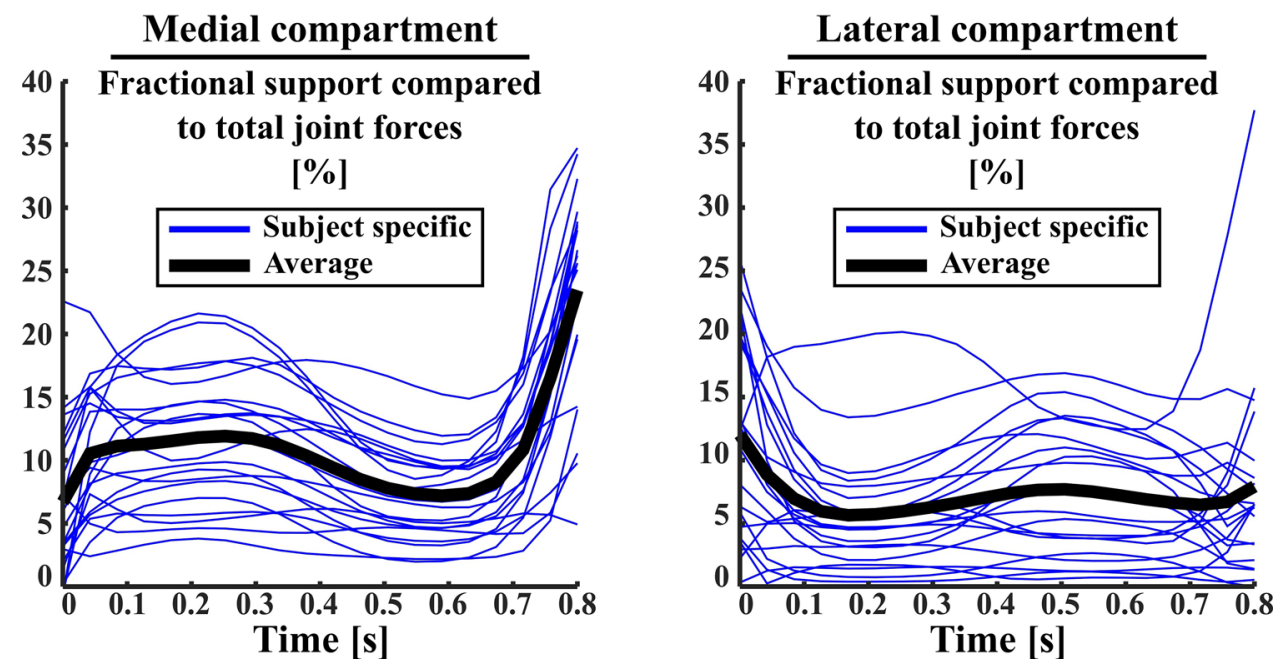

FIGURE 4. Forces through the lateral (left) and medial (right) meniscus as a function of stance phase of the gait cycle.

\section{Degenerated volumes $\left[\mathrm{mm}^{3}\right]$}

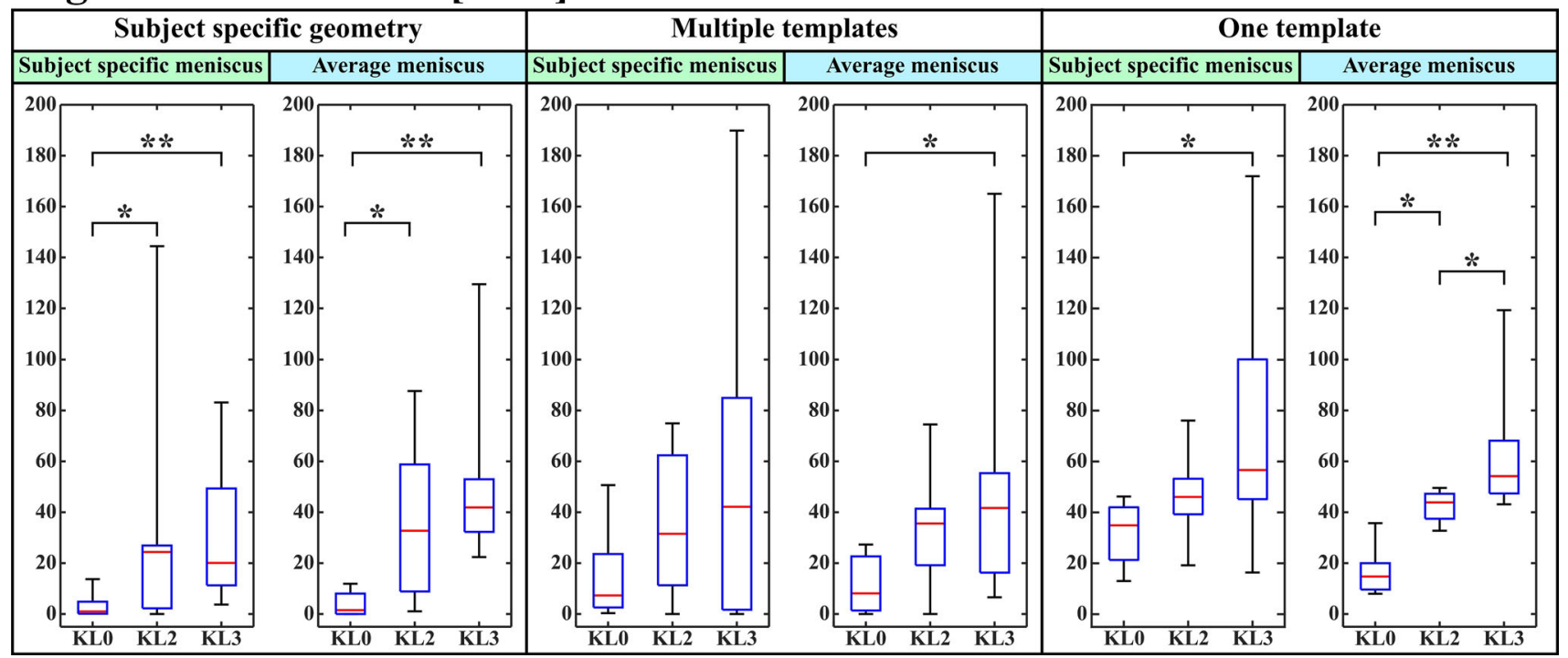

FIGURE 5. Predicted degenerated volumes in patient-specific (left), multiple template (middle) and one template (right) approaches for each KL group. Patient-specific and template approaches were utilized with both subject-specific and average meniscal support. Crosslines (-) represent median values. Statistical significances between different $\mathrm{KL}$ groups were evaluated by using Kruskal-Wallis $H$ test, ${ }^{*} p<0.05$ and ${ }^{* \star} p<0.01$.

and average meniscus forces, statistically significant differences were found between KL0 and KL2 groups and between KL0 vs. KL3 groups, but no differences were found between KL2 and KL3 groups (Fig. 5, left). In the multiple template approach with subjectspecific meniscus support, statistically significant differences were not found between any groups. When the effect of meniscus support was changed to average, statistically significant differences were found between KL0 and KL3 groups (Fig. 5, middle). In the one template approach with subject-specific meniscus sup- port, statistically significant differences were found only between KL0 and KL3 groups. Interestingly, when the effect of meniscus support was changed to average, statistically significant differences were found between all groups (Fig. 5, right).

In terms of statistical differences between different $\mathrm{KL}$ groups, the receiving operating characteristic (ROC)-curves and area under the curves (AUC) produced identical results compared to what were obtained from Kruskal-Wallis $H$ test (Figs. 5 vs. 6). Based on the ROC analysis, KL0 and KL3 groups 
Receiving Operating Characteristics (ROC) -curves and Area Under Curve (AUC) -values for the degenerated volumes

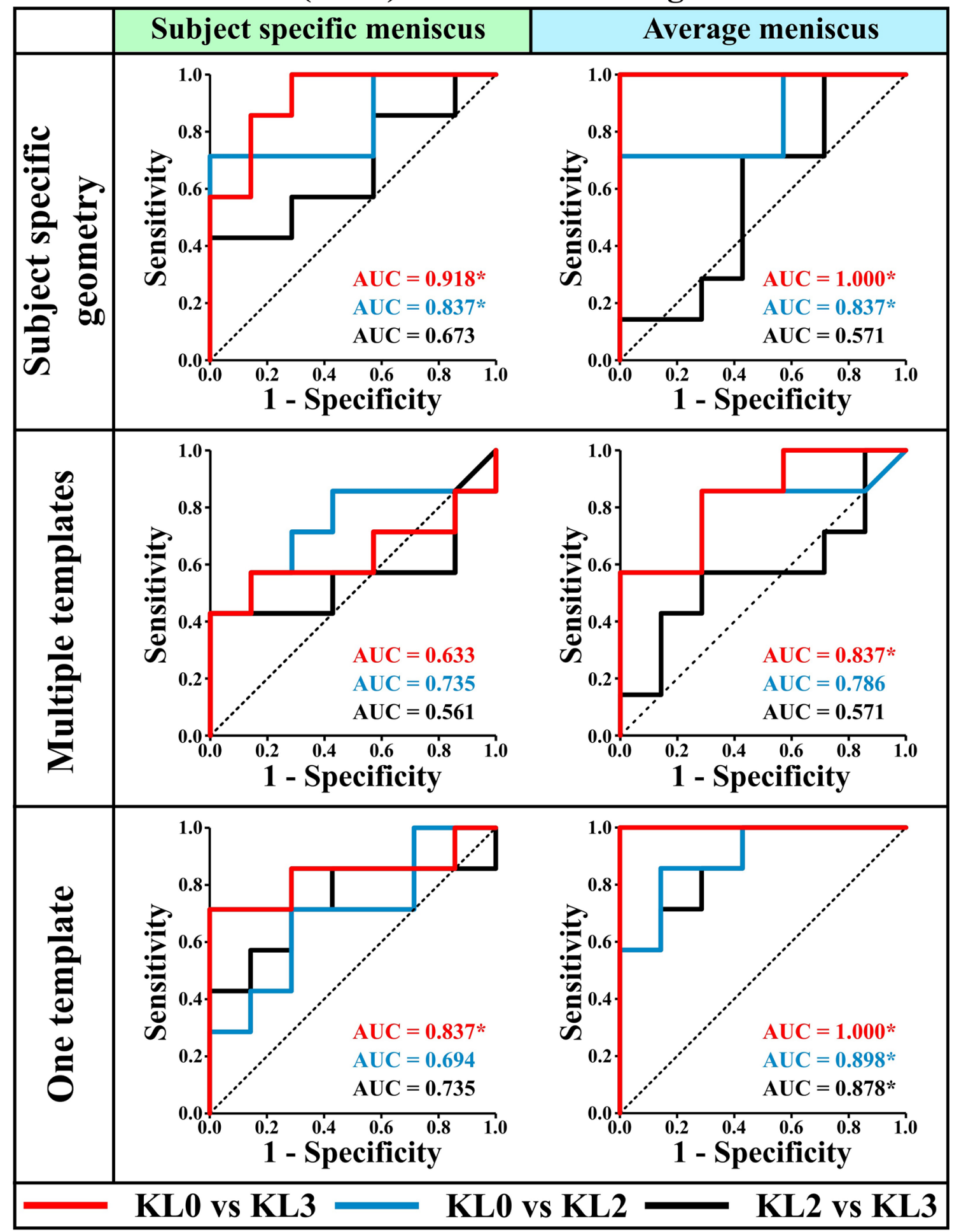

FIGURE 6. Receiving operating characteristics (ROC)-curves and area under curve (AUC)-values for the volume of degenerated elements in each approach. ${ }^{*} p<0.05$ and ${ }^{* \star} p<0.01$. 
were separated perfectly from each other $(\mathrm{AUC}=1$, $p<0.01)$ in the models with subject-specific geometries and one template with average meniscus support (Fig. 6, top-right and bottom-right). In these models, statistical differences between KL0 and KL2 groups was also observed (AUC $>0.8, p<0.05$ ). Interestingly, statistically significant differences between KL2 and KL3 groups were seen only in the template approach with one template $(\mathrm{AUC}=0.878$, $p<0.05)$.

\section{DISCUSSION}

In the current study, the ability of a template based modeling approach to predict development of knee osteoarthritis during physiologically relevant loading conditions (simplified gait load) was tested. The model results were compared against patient-specific models and experimental follow-up radiographic KL grades of 21 subjects. Both subject-specific and template- or population-based models (excluding the multiple template approach with subject-specific meniscus consideration) were statistically able to separate healthy subjects from those whom KL grades were increased from 0 to 3 , especially when the average consideration of forces through the meniscus (meniscus support) was considered. Surprisingly, the approach with one template (average geometry) and the average meniscus support was statistically able to separate all KL grade groups from each other.

We initially suspected that the subject-specific approach would provide best predictions to classify studied subjects into correct KL grade groups. However, in terms of statistical significance, the simplest method with one template showed the most promising results. The reason for this may be related to differences in implemented loads. In the subject-specific approaches, free varus/valgus rotation can lead to cases where either one of the compartments is more loaded than the other, while the template approach considered $50 \%$ of the total joint loads throughout the loading. Here, in the subject-specific approach, there were two subjects in the KL3 group, where the peak forces through the medial compartment were considerably lower than those in the KL2 group. This may explain why the statistical difference was not found between the KL2 and KL3 groups, though the mean forces through the medial compartment during the entire stance phase remained similar between these groups.

Due to similar BMIs of the subjects in KL2 and KL3 grade groups, and the same material and boundary conditions, the only major difference between these subjects was the knee geometry. There- fore, different volumetric degenerations obtained for these groups suggest that geometrical variation plays an important role to predict the onset and development of knee OA. This same result was obtained for all studied approaches (patient- and population-specific), though some differences in the statistical analysis were seen, suggesting that the used anatomical dimensions in the template approach may be sufficient when predicting OA. This is a very promising result, since the main bottleneck in the simulations of large number of subjects is time required in segmentation, meshing, implementing boundary conditions and material, and model simulation. In the subject-specific approach, this entire process from the model generation till prediction took at minimum of 1 week per subject. Sometimes it can take weeks or months to get results. The template approach was able to decrease this time to $\sim 2 \mathrm{~min}$. Furthermore, the working reliability in the template approach was excellent because the used FE mesh was tested thoroughly beforehand. Nonetheless, it is still too early to say which one of the presented methods is the best due to the subject number. More simulations are needed with more subjects.

Interestingly, by considering the average meniscus support with the subject-specific cartilage geometries, model predictions did not change from the statistical point of view. Instead, in multiple template and one template approaches, consideration of the average meniscus support helped to separate all KL grade groups from each other. True validation of the meniscal support is obviously impossible to conduct in vivo, and for instance pretensions of the meniscal attachments affect the distribution of knee joint loads. ${ }^{14}$ Furthermore, small errors (error of one pixel in MRI) in meniscus segmentation may lead to overestimated or underestimated tissue thicknesses, which may substantially influence the meniscus support. Nonetheless, eliminating the possible influence of these uncertainties, and using only one meniscus supporting force (in bodyweight) in each model, produced promising results and could provide a simple solution to consider meniscus.

Meniscal support forces varied a lot between subjects. Current literature knows very few experimental studies related to meniscal support during physiological loading conditions. In 2014, a group of researchers was able to attach 5 knee joints from cadavers into a modified knee joint stimulator and to measure forces through meniscus under loadings suspected to occur during normal walking. ${ }^{13}$ In that study, forces through the lateral meniscus were constantly $\sim 50 \%$ of the total forces recorded in the lateral compartment, whereas forces through the medial meniscus were between $\sim 25$ and $\sim 50 \%$ of the total forces recorded in the medial compartment. In the current study, only in some sub- 
jects such high meniscal support was achieved, whereas the average forces through the lateral and medial meniscus varied between $\sim 10-25$ and $\sim 15-45 \%$ of the total forces, respectively. Recently, forces through the meniscus during gait were evaluated for two subjects using multibody simulation with different consideration of zero-load length for meniscal attachments. ${ }^{14} \mathrm{In}$ that study, one of the results was that the medial meniscus transfers more loads compared to the lateral meniscus during the stance phase of gait, and the amount of load transmission may be influenced by the subject geometry. This result is consistent with the results of the current study.

In earlier studies, ${ }^{3,22}$ it has been shown that the subject-specific joint loading is important to consider when investigating force distributions between the lateral and medial compartments and forces in the patellofemoral contact. For instance, forces through the medial compartment may vary from 55 to $88 \%$ and from 44 to $84 \%$ of the total joint forces during the first and second peak forces of gait, respectively. ${ }^{22}$ This subject-specific variation in the force distributions may be explained mainly by the geometrical differences and hip-knee-ankle (HKA) angle. ${ }^{22}$ In the current study, in the subject-specific approach, free varus/valgus rotation produced $\sim 50 \% / 50 \%$ force distribution between medial and lateral compartments and only small variations in this force balance through entire loading. Though, as discussed above, it should be noted that even small variation in this force balance may slightly influence the predicted degeneration volume. This distribution matches adequately with the selected experimental measurements. ${ }^{22}$ This was also the motivation to use exactly $50 \%$ of the total reaction force in the medial compartment of the template approach. Even though subject-specific gait data was unavailable and thus could not be implemented, surprisingly good predictions for the development of knee OA were obtained.

In addition to mechanical overloading and aging, it has been shown that the risk for the onset and development of knee OA is also strongly correlated for instance with knee alignment, gender, occupational hazard (long-term kneeling/squatting), inflammation due to joint injuries or overload, and genetic factors. ${ }^{10,11}$ These factors were not considered in the current study. To consider the above-mentioned parameters, (i) they should be known, (ii) subject number should be increased considerably, and/or (iii) they should be studied first in controlled in vitro and/or animal model experiments.

The choice to use the relatively complex material model for cartilage is justified by its ability to distinguish between different tissue constituents (collagen, proteoglycan, fluid) and to model local and con- stituent-specific tissue degradation. ${ }^{26,31,32}$ Simplification of the material model would not enable this. However, especially as we compare the modeling results against KL grades, simpler modeling approaches might produce the same conclusions. In order to make clear conclusions about the needed level of model sophistication to provide adequate results, different modeling approaches should to be compared in the future.

The presented results suggest that a population-, template-based knee joint models may be applicable to predict OA and classify subjects into correct groups. In the future, the presented workflow should be tested against larger subject groups. This could provide a fast clinical tool for prediction of osteoarthritis and simulation of interventions.

\section{ACKNOWLEDGMENTS}

Open access funding provided by University of Eastern Finland (UEF) including Kuopio University Hospital. This project has received funding from the European Research Council (ERC) under the European Union's Horizon 2020 research and innovation programme (Grant Agreement No 755037); the Academy of Finland (Grant Numbers 286526, 305138); Doctoral Programme in Science, Technology and Computing, University of Eastern Finland; and Sigrid Juselius Foundation. CSC-IT Center for Science Ltd, Finland is acknowledged for providing the finite-element modeling software and OAI for providing the MRI and Kellgren-Lawrence (KL) data from the public use dataset (http://oai.epi-ucsf. org/). The OAI is a public-private partnership comprised of five contracts (N01-AR-2-2258; N01-AR-22259; N01-AR-2-2260; N01-AR-2-2261; N01-AR-22262) funded by the National Institutes of Health, a branch of the Department of Health and Human Services, and conducted by the OAI Study Investigators. Private funding partners include Merck Research Laboratories; Novartis Pharmaceuticals Corporation, GlaxoSmithKline; and Pfizer, Inc. Private sector funding for the OAI is managed by the Foundation for the National Institutes of Health. This manuscript was prepared using an OAI public use data set and does not necessarily reflect the opinions or views of the OAI investigators, the NIH, or the private funding partners.

\section{OPEN ACCESS}

This article is distributed under the terms of the Creative Commons Attribution 4.0 International Li- 
cense (http://creativecommons.org/licenses/by/4.0/), which permits unrestricted use, distribution, and reproduction in any medium, provided you give appropriate credit to the original author(s) and the source, provide a link to the Creative Commons license, and indicate if changes were made.

\section{REFERENCES}

${ }^{1}$ Adouni, M., A. Shirazi-Adl, and R. Shirazi. Computational biodynamics of human knee joint in gait: from muscle forces to cartilage stresses. J. Biomech. 45:21492156, 2012.

${ }^{2}$ Ahn, C., T. D. Bui, Y. W. Lee, J. Shin, and H. Park. Fully automated, level set-based segmentation for knee MRIs using an adaptive force function and template: data from the osteoarthritis initiative. Biomed. Eng. Online 15:99, 2016.

${ }^{3}$ Akbarshahi, M., J. W. Fernandez, A. G. Schache, and M. G. Pandy. Subject-specific evaluation of patellofemoral joint biomechanics during functional activity. Med. Eng. Phys. 36:1122-1133, 2014.

${ }^{4}$ Ali, A. A., M. D. Harris, S. Shalhoub, L. P. Maletsky, P. J. Rullkoetter, and K. B. Shelburne. Combined measurement and modeling of specimen-specific knee mechanics for healthy and ACL-deficient conditions. J. Biomech. 57:117124, 2017.

${ }^{5}$ Baliunas, A. J., D. E. Hurwitz, A. B. Ryals, A. Karrar, J. P. Case, J. A. Block, and T. P. Andriacchi. Increased knee joint loads during walking are present in subjects with knee osteoarthritis. Osteoarthr. Cartil. 10:573-579, 2002.

${ }^{6}$ Bedi, A., N. H. Kelly, M. Baad, A. J. Fox, R. H. Brophy, R. F. Warren, and S. A. Maher. Dynamic contact mechanics of the medial meniscus as a function of radial tear, repair, and partial meniscectomy. J. Bone Joint Surg. Am. 92:1398-1408, 2010.

${ }^{7}$ Bedi, A., N. Kelly, M. Baad, A. J. Fox, Y. Ma, R. F. Warren, and S. A. Maher. Dynamic contact mechanics of radial tears of the lateral meniscus: implications for treatment. Arthroscopy 28:372-381, 2012.

${ }^{8}$ Bergmann, G., A. Bender, F. Graichen, J. Dymke, A. Rohlmann, A. Trepczynski, M. O. Heller, and I. Kutzner. Standardized loads acting in knee implants. PLOS ONE 9:e86035, 2014.

${ }^{9}$ Brehler, M., G. Thawait, W. Shyr, J. Ramsay, J. H. Siewerdsen, and W. Zbijewski. Atlas-based automatic measurements of the morphology of the tibiofemoral joint. Proc. SPIE Int. Soc. Opt. Eng. 2017. https://doi.org/10. $1117 / 12.2255566$.

${ }^{10}$ Coggon, D., P. Croft, S. Kellingray, D. Barrett, M. McLaren, and C. Cooper. Occupational physical activities and osteoarthritis of the knee. Arthritis Rheum. 43:14431449, 2000.

${ }^{11}$ Englund, M. The role of biomechanics in the initiation and progression of OA of the knee. Best Pract. Res. Clin. Rheumatol. 24:39-46, 2010.

${ }^{12}$ Fukubayashi, T., and H. Kurosawa. The contact area and pressure distribution pattern of the knee. A study of normal and osteoarthrotic knee joints. Acta Orthop. Scand. 51:871879, 1980.

${ }^{13}$ Gilbert, S., T. Chen, I. D. Hutchinson, D. Choi, C. Voigt, R. F. Warren, and S. A. Maher. Dynamic contact mechanics on the tibial plateau of the human knee during activities of daily living. J. Biomech. 47:2006-2012, 2014.

${ }^{14}$ Guess, T. M., S. Razu, H. Jahandar, and A. Stylianou. Predicted loading on the menisci during gait: the effect of horn laxity. J. Biomech. 48:1490-1498, 2015.

${ }^{15}$ Halonen, K. S., M. E. Mononen, J. S. Jurvelin, J. Toyras, and R. K. Korhonen. Importance of depth-wise distribution of collagen and proteoglycans in articular cartilage: a 3D finite element study of stresses and strains in human knee joint. J. Biomech. 46:1184-1192, 2013.

${ }^{16}$ Haq, R., R. Aras, D. A. Besachio, R. C. Borgie, and M. A. Audette. 3D lumbar spine intervertebral disc segmentation and compression simulation from MRI using shape-aware models. Int. J. Comput. Assist. Radiol. Surg. 10:45-54, 2015.

${ }^{17}$ Horisberger, M., R. Fortuna, V. Valderrabano, and W. Herzog. Long-term repetitive mechanical loading of the knee joint by in vivo muscle stimulation accelerates cartilage degeneration and increases chondrocyte death in a rabbit model. Clin. Biomech. 28:536-543, 2013.

${ }^{18}$ Jones, B., C. T. Hung, and G. Ateshian. Biphasic analysis of cartilage stresses in the patellofemoral joint. J. Knee Surg. 29:92-98, 2016.

${ }^{19}$ Kazemi, M., and L. P. Li. A viscoelastic poromechanical model of the knee joint in large compression. Med. Eng. Phys. 36:998-1006, 2014.

${ }^{20}$ Kempson, G. E. Relationship between the tensile properties of articular cartilage from the human knee and age. Ann. Rheum. Dis. 41:508-511, 1982.

${ }^{21}$ Kim, T. K., M. Phillips, M. Bhandari, J. Watson, and R. Malhotra. What differences in morphologic features of the knee exist among patients of various races? A systematic review. Clin. Orthop. Relat. Res. 475:170-182, 2017.

${ }^{22}$ Kutzner, I., A. Bender, J. Dymke, G. Duda, P. von Roth, and G. Bergmann. Mediolateral force distribution at the knee joint shifts across activities and is driven by tibiofemoral alignment. Bone Joint J. 99:779-787, 2017.

${ }^{23}$ Kutzner, I., B. Heinlein, F. Graichen, A. Bender, A. Rohlmann, A. Halder, A. Beier, and G. Bergmann. Loading of the knee joint during activities of daily living measured in vivo in five subjects. J. Biomech. 43:2164-2173, 2010.

${ }^{24}$ Lee, J. G., S. Gumus, C. H. Moon, C. K. Kwoh, and K. T. Bae. Fully automated segmentation of cartilage from the MR images of knee using a multi-atlas and local structural analysis method. Med. Phys. 41:092303, 2014.

${ }^{25}$ Linden, A. Measuring diagnostic and predictive accuracy in disease management: an introduction to receiver operating characteristic (ROC) analysis. J. Eval. Clin. Pract. 12:132-139, 2006.

${ }^{26}$ Liukkonen, M. K., M. E. Mononen, O. Klets, J. P. Arokoski, S. Saarakkala, and R. K. Korhonen. Simulation of subject-specific progression of knee osteoarthritis and comparison to experimental follow-up data: data from the osteoarthritis initiative. Sci. Rep. 7:9177, 2017.

${ }^{27}$ Liukkonen, M. K., M. E. Mononen, P. Tanska, S. Saarakkala, M. T. Nieminen, and R. K. Korhonen. Application of a semi-automatic cartilage segmentation method for biomechanical modeling of the knee joint. Comput. Methods Biomech. Biomed. Eng. 20:1453-1463, 2017.

${ }^{28}$ Liukkonen, M. K., M. E. Mononen, P. Vartiainen, P. Kaukinen, T. Bragge, J. S. Suomalainen, M. K. H. Malo, S. Venesmaa, P. Kakela, J. Pihlajamaki, P. A. Karjalainen, J. P. Arokoski, and R. K. Korhonen. Evaluation of the effect of bariatric surgery-induced weight loss on knee gait 
and cartilage degeneration. J. Biomech. Eng. 25:23, 2018. h ttps://doi.org/10.1115/1.4038330.

${ }^{29}$ Loeser, R. F., J. A. Collins, and B. O. Diekman. Ageing and the pathogenesis of osteoarthritis. Nat. Rev. Rheumatol. 12:412-420, 2016

${ }^{30}$ Miller, R. H., W. B. Edwards, S. C. Brandon, A. M. Morton, and K. J. Deluzio. Why don't most runners get knee osteoarthritis? A case for per-unit-distance loads. Med. Sci. Sports Exerc. 46:572-579, 2014.

${ }^{31}$ Mononen, M. E., P. Tanska, H. Isaksson, and R. K. Korhonen. A novel method to simulate the progression of collagen degeneration of cartilage in the knee: data from the osteoarthritis initiative. Sci. Rep. 6:21415, 2016.

${ }^{32}$ Mononen, M. E., P. Tanska, H. Isaksson, and R. K. Korhonen. New algorithm for simulation of proteoglycan loss and collagen degeneration in the knee joint: data from the osteoarthritis initiative. J. Orthop. Res. 2017. https://d oi.org/10.1002/jor.23811.

${ }^{33}$ Rodriguez-Vila, B., P. Sanchez-Gonzalez, I. Oropesa, E. J. Gomez, and D. M. Pierce. Automated hexahedral meshing of knee cartilage structures - application to data from the osteoarthritis initiative. Comput. Methods Biomech. Biomed. Eng. 20:1543-1553, 2017.

${ }^{34}$ Sadeghi, H., D. E. Shepherd, and D. M. Espino. Effect of the variation of loading frequency on surface failure of bovine articular cartilage. Osteoarthr. Cartil. 23:2252-2258, 2015.

${ }^{35}$ Schwarzkopf, R., A. Bauer, A. Chaurasia, A. M. Hall, D. Zurakowski, and R. D. Scott. Distal femoral aspect ratios throughout childhood: an MRI study of normative data and sex comparisons. J. Pediatr. Orthop. B. 25:241-244, 2016.

${ }^{36}$ Seedhom, B. B. Conditioning of cartilage during normal activities is an important factor in the development of osteoarthritis. Rheumatology 45:146-149, 2006.

${ }^{37}$ Shan, L., C. Charles, and M. Niethammer. Automatic multi-atlas-based cartilage segmentation from knee MR images. Proc. IEEE Int. Symp. Biomed. Imaging 2012:10281031, 2012.

${ }^{38}$ Shane Anderson, A., and R. F. Loeser. Why is osteoarthritis an age-related disease? Best Pract. Res. Clin. Rheumatol. 24:15-26, 2010.

${ }^{39}$ Vaziri, A., H. Nayeb-Hashemi, A. Singh, and B. A. Tafti. Influence of meniscectomy and meniscus replacement on the stress distribution in human knee joint. Ann. Biomed. Eng. 36:1335-1344, 2008.

${ }^{40}$ Venalainen, M. S., M. E. Mononen, J. Salo, L. P. Rasanen, J. S. Jurvelin, J. Toyras, T. Viren, and R. K. Korhonen. Quantitative evaluation of the mechanical risks caused by focal cartilage defects in the knee. Sci. Rep. 6:37538, 2016.

${ }^{41}$ Wilson, W., C. C. van Donkelaar, B. van Rietbergen, K. Ito, and R. Huiskes. Stresses in the local collagen network of articular cartilage: a poroviscoelastic fibril-reinforced finite element study. J. Biomech. 37:357-366, 2004.

${ }^{42}$ Wilson, W., C. C. van Donkelaar, B. van Rietbergen, K. Ito, and R. Huiskes. Erratum to "Stresses in the local collagen network of articular cartilage: a poroviscoelastic fibril-reinforced finite element study"[J Biomech 37 (2004) 357-366] and "A fibril-reinforced poroviscoelastic swelling model for articular cartilage' [J Biomech 38 (2005) 11951204]. J. Biomech. 38:2138-2140, 2005.

${ }^{43}$ Wise, B. L., J. Niu, M. Yang, N. E. Lane, W. Harvey, D. T. Felson, J. Hietpas, M. Nevitt, L. Sharma, J. Torner, C. E. Lewis, Y. Zhang, and Multicenter Osteoarthritis (MOST) Group. Patterns of compartment involvement in tibiofemoral osteoarthritis in men and women and in whites and African Americans. Arthritis Care. Res. 64:847-852, 2012.

${ }^{44}$ Zhou, Z. Y., Y. K. Liu, H. L. Chen, and F. Liu. Body mass index and knee osteoarthritis risk: a dose-response metaanalysis. Obesity 22:2180-2185, 2014.

${ }^{45}$ Zielinska, B., and T. L. Donahue. 3D finite element model of meniscectomy: changes in joint contact behavior. $J$. Biomech. Eng. 128:115-123, 2006. 